\title{
7 Herstellerverzeichnis
}

Hinweis: Die im Buch verwendeten Abkürzungen sind jeweils dem Herstellernamen vorangestellt.

ADV: ADVANCIS Medical Deutschland GmbH

Rütersbarg 48

D-22529 Hamburg

Tel.: + 494087408062

E-Mail: info@advancismedical.de

Web: www.advancismedical.de

AKT: Aktivmed GmbH

Oldenburger Str. 17

D-48429 Rheine

Tel.: + 498001227777

E-Mail: info@aktivmed.de

Web: www.aktivmed.de

BBR: B. Braun Melsungen AG

Carl-Braun-Str. 1

D-34212 Melsungen

Tel.: + 495661710

E-Mail: info@bbraun.com

Web: www.bbraun.de

BIC: Biocell Gesellschaft für Biotechnologie mbH

Hauptstr. 60

D-53804 Much

Tel.: + 4922459037040

E-Mail: info@biocell.de

Web: www.biocell.de

BIM: Biomonde $\mathrm{GmbH}$

Kiebitzhörn 33-35

D-22885 Barsbüttel

Tel.: + 49406710570

E-Mail: info@biomonde.de

Web: www.biomonde.de 
BIV: Biovision

Am Vogelherd 52

D-98693 Ilmenau

Tel.: + $4936776407-10$

E-Mail: info@biovision.de

Web: www.biovision.de

BSN: BSN medical GmbH \& Co. KG

Quickbornstr. 24

D-20253 Hamburg

Tel.: + $49404909-909$

E-Mail: info@bsnmedical.de

Web: www.bsnmedical.de/bsn-medical-deutschland.html

COL: Coloplast $\mathrm{GmbH}$

Kuehnestr. 75

D-22045 Hamburg

Tel.: + $4940669807-0$

E-Mail: service@coloplast.com

Web: www.coloplast.de

CON: ConvaTec Germany GmbH

Radlkoferstr. 2

D-81373 München

Tel.: + 49897866200

E-Mail: convatec.servicede@convatec.com

Web: www.convatec.de

COV: Covidien Deutschland GmbH

Gewerbepark 1

D-93333 Neustadt an der Donau

Tel.: + 4994459590

E-Mail: deutschland@medtronic.com

Web: www.medtronic.com/de-de/index.html

CRA: Crawford Healthcare GmbH

Medizinpark 1

D-83626 Valley

Tel.: + 4980244686820

E-Mail: info@crawfordhealthcare.de

Web: www.crawfordhealthcare.de 
CUR: Curea Medical

Rudolf-Diesel-Str. 6

D-37308 Heilbad Heligenstadt

Tel.: + 49360719009500

E-Mail: info@curea-medical.de

Web: www.curea-medical.de

DRA: Draco, Dr. Ausbüttel \& Co GmbH

Herdecker Str. 9-15

D-58453 Witten-Annen

Tel.: + 49230295 666-285

E-Mail: kontakt@draco.de

Web: www.draco.de

FLE: Flen Pharma

Königsallee 92A

D-40212 Düsseldorf

Tel.: + 4921154039819

E-Mail: info_de@flenhealth.com

Web: www.flenhealth.com

FRE: Fresenius Kabi

Else-Kröner-Str. 1

D-61352 Bad Homburg

Tel.: + 496172 686-0

E-Mail: communication@fresenius-kabi.com

Web: www.fresenius-kabi.com

HAR: Paul Hartmann AG

Paul-Hartmann-Str. 12

D-89522 Heidenheim

Tel.: + 497321360

E-Mail: info@hartmann.info

Web: https://hartmann.info/de-de

HEX: Hexal AG

Industriestr. 25

D-83607 Holzkirchen

Tel.: + 498024 908-0

E-Mail: service@hexal.com

Web: www.hexal.de 
HOL: Hollister

Riesstr. 25

D-80992 München

Tel.: + $4989992886-0$

E-Mail: beratungsteam@hollister.com

Web: www.hollister.de

i3: i3 Membrane $\mathrm{GmbH}$

Theodorstr. $41 \mathrm{P}$

D-22761 Hamburg

Tel.: + $49402576748-0$

E-Mail: info@i3membrane.de

Web: www.i3membrane.de/de/

INT: Integralife $\mathrm{GmbH}$

Halskestr. 9

D-40880 Ratingen

Tel.: + 4921025 535-6200

E-Mail: custsvcgermany@integralife.com

Web: www.integralife.com/home

J\&J: Johnson \& Johnson Wound Management Ethicon GmbH

Robert-Koch-Str. 1

D-22851 Norderstedt

Tel.: + 494052973200

E-Mail: endolnfo@lts.JnJ.com

Web: www.jnjmedicaldevices.com/de-DE

KBE: Karl Beese [GmbH Co.]

Großer Kamp 12-14

D-22885 Barsbüttel

Tel.: + 49406706550

E-Mail: info@karl-beese.de

Web: www.karl-beese.de

KCI: KCI Medizinprodukte GmbH

Am Klingenweg 10

D-65396 Wallief

Tel.: + 498007833524

E-Mail: kundenservice@acelity.com

Web: www.kci.de 
KER: Kerecis AG

Webereistr. 61

CH-8134 Adliswil

Tel.: + 414991566

E-Mail: support@1wound.info

Web: www.kerecis.com

LIG: LIGAMED medical Produkte GmbH

Pfannenstielstr. 14

D-90556 Cadolzburg

Tel.: + 4991032046

E-Mail: info@ligamed.de

Web: www.ligamed.de

L\&R: Lohmann \& Rauscher GmbH \& Co. Kg

Westerwaldstr. 4

D-56579 Rengsdorf

Tel.: + 492634990

E-Mail: info@de.LRmed.com

Web: www.lohmann-rauscher.com

MBP: Medical Biomaterial Products

Lederstr. 7

D-19306 Neustadt-Glewe

Tel.: + 4938757 509-0

E-Mail: info@mbp-gmbh.de

Web: www.mbp-gmbh.de

MDH: Medihoney

Jäckstr. 29

D-96052 Bamberg

Tel.: + 499515107600

E-Mail: info@apofit-gmbh.de

Web: www.apofit-gmbh.de

MED: Mediset Clinical Products $\mathrm{GmbH}$

Spenglerallee 12-14

D-04442 Zwenkau

Tel.: + 49210436832

E-Mail: info@mediset.de

Web: www.mediset.de 
MET: Meditrade GmbH

Medipark 1

D-83088 Kiefersfelden

Tel.: + $4980339760-0$

E-Mail: info@meditrade.de

Web: www.meditrade.de

MÖL: Mölnlycke Health Care GmbH

Max-Planck-Str. 15

D-40699 Erkrath

Tel.: + 49211920880

E-Mail: info.de@molnlycke.com

Web: www.molnlycke.de

MUN: Mundipharma

De-Saint-Exupéry-Str. 10

D-60549 Frankfurt am Main

Tel.: + 4969506 029-000

E-Mail: info@mundipharma.de

Web: www.mundipharma.de

NOB: NOBAMED Paul Danz AG

Höltkenstr. 1-5

D-58300 Wetter

Tel.: + 4923357 609-0

E-Mail: info@nobamed-ag.com

Web: www.nobamed-ag.com

POL: PolyMedics

Heerweg 15 D

D-73770 Denkendorf

Tel.: + 497117195000

E-Mail: info@polymedics.de

Web: www.suprathel.com

ROB: ROBIN WOUND GmbH

Nell-Breuning-Allee 6

D-66115 Saarbrücken

Tel.: + 496819597190

Web: www.robin-wound.com 
ROG: ROGG Verbandstoffe GmbH

Oberndorfer Str. 7

D-85777 Fahrenzhausen

Tel.: + 49813393200

E-Mail: shop@rogg-verbandstoffe.de

Web: www.rogg-webshop.de

SNA: sanaFactur GmbH

Saturnring 4

D-32130 Enger

Tel.: + 4952249992490

E-Mail: info@sanafactur.com

Web: www.sanafactur.com

SNO: Sanofi

Potsdamer Str. 8

D-10785 Berlin

Tel.: + 493025752000

E-Mail: medinfo.de@sanofi.com

Web: www.sanofi.de

S\&M: Schülke \& Mayr

Robert-Koch-Str. 2

D-22851 Norderstedt

Tel.: + 4940521000

E-Mail: info@schuelke.com

Web: www.schuelke.com

SER: Serag-Wiessner

Zum Kugelfang 8-12

D-95119 Naila

Tel.: + 4992829370

E-Mail: info@serag-wiessner.de

Web: www.serag-wiessner.de

S\&N: Smith \& Nephew GmbH

Max-Planck-Str. 1-3

D-34253 Lohfelden

Tel.: + 49408797440

E-Mail: info@smith-nephew.com

Web: www.smith-nephew.de 
SÖR: Söhring GmbH

Justus-von-Liebig-Ring 2

D-25451 Quickborn

Tel.: + $4941066100-0$

E-Mail: info@soehring.com

Web: www.soehring.de

SYS: Systagenix

Hagenauer Str. 47

D-65203 Wiesbaden

Tel.: + $4980051670-00$

E-Mail: info@gesundheitsgmbh.de

Web: www.gesundheitsgmbh.de

TRI: Trigocare International $\mathrm{GmbH}$

In den Wiesen 3

D-51674 Wiehl

Tel.: + 492262727010

Web: www.trigocare.com

URG: Urgo GmbH

Justus-von-Liebig-Str. 16

D-66280 Sulzbach

Tel.: + 496897 579-0

E-Mail: info@urgo.de

Web: www.urgo.de 